\title{
Diagnóstico genético preimplantación: mirando al futuro.
}

\author{
Juan Luis Giraldo, Antonia Habana ${ }^{1 *}$; Paulo Serafini**; David Olive ${ }^{* * *}$
}

\section{RESUMEN}

El diagnóstico genético preimplantación (PGD) es en el momento la forma más temprana de diagnóstico prenatal. En la década de los noventa su carácter experimental comienza a dar paso a su aplicación clínica. Se han reportado ya numerosos casos de nacidos vivos sometidos a PGD para la detección de enfermedades ligadas al sexo, enfermedades monogénicas y aneuploidías. Además su papel en la optimización del resultado de las técnicas de reproducción asistida está en estudio y parece promisorio, especialmente en el grupo de mujeres de mayor edad. Actualmente se utilizan la hibridización in situ fluorescente (FISH) y la reacción en cadena de polimerasa (PCR) como principales herramientas en el análisis del material genético del embrión. La obtención de este material se ha logrado mediante la biopsia de cuerpo polar, de blastómera y de trofoectodermo. En el seguimiento a corto y mediano plazo el PGD ha demostrado ser una técnica eficiente y segura, pero se carece aún de estudios con seguimiento a largo plazo que corroboren estos aspectos. Avances en el uso del PGD que faciliten su aplicación y disminuyan su costo serán responsables de una propagación en el uso de este método de diagnóstico prenatal en los próximos años.

PALABRAS CLAVES: Diagnóstico Genético Preimplantación(PGD)/FISH/PCR/enfermedades monogénicas/enfermedades ligadas al cromosoma $X$ / aneuploidías / fertilización in vitro.

\section{ABSTRACT}

Preimplantation genetic diagnosis (PGD) is actually the earliest of all prenatal diagnosis. In this decade there has been a gradual increased use of the technique from a research tool to one that can be clinically applicable. Presently there has been numerous cases reported of newborns that have undergone this diagnostic test for detection of $X$-linked diseases, monogenic diseases and aneuploidies. Furthermore, its' use for the improvement in outcome of assisted reproductive techniques appears promising, especially in older women. Fluorescent in situ hybridization (FISH) and polymerase chain reaction (PCR) are the most used techniques to analyze the embryo genetic material. For this purpose embryo biopsy has been done to obtain polar bodies, blastomeres and trophectoderm cells. With short term follow up, PGD appears to be an efficient and safe technique, but long term follow up studies are still needed. Advance in PGD techniques that simplifies its' implementation and decreases its' cost will account for its' widespread use in the years to come.

KEY WORDS: Preimplantation genetic diagnosis (PGD) / FISH / PCR / IVF / monogenic diseases / X-linked diseases/aneuploidies

\section{Introducción}

El diagnóstico genético preimplantación (PGD) es un procedimiento joven. La investigación en este campo se inicio en 1980 como resultado de la presión de parejas con abortos terapeúticos repetidos debido a alteraciones genéticas diagnosticadas inútero, parejas con objeciones morales al aborto terapeútico y parejas con riesgo de transmitir a sus hijos enfermedades de origen genético. El primer reporte de un nacido vivo luego de ser sometido al PGD se dió en 1990 (1) cuando con el uso de reacción en cadena de polimerasa (PCR) se amplificaron

Fellow, División Medicina Reproductiva e Infertilidad, Departamento de Obstetricia y Ginecología, Universidad de Yale, USA. Profesor Asociado, División de Medicina Reproductiva e Infertilidad, Departamento de Obstetricia y Ginecología, Universidad de Yale, USA.

Director de la División de Medicina Reproductiva e Infertilidad, Departamento de Obstetricia y Ginecología, Universidad de Yale, USA. secuencias específicas del cromosoma $\mathrm{Y}$ en varios embriones, permitiendo la selección y transferencia de embriones femeninos para evitar enfermedades hereditarias ligadas al cromosoma $\mathrm{X}$. El desarrollo de esta modalidad de diagnóstico genético ha llevado a que su aplicación clínica sea cada vez más relevante e inclusive a que se considere su uso rutinario en cierto grupo de pacientes (2).

El PGD se erige como una forma muy temprana de diagnóstico prenatal para parejas con riesgo de transmitir a sus hijos enfermedades de origen genético. Es una buena opción frente a las demás modalidades de diagnóstico genético prenatal que incluyen la biopsia de vellosidades coriónicas y la amniocentesis, a pesar de que actualmente la mayor parte de los centros utilizan estas últimas como método para confirmar el diagnóstico del PGD, dado el carácter aún experimental del mismo. Además, evita el trastorno generado por la terminación del embarazo en el primer y segundo trimestre cuando se diagnostica una anomalía genética en el feto mediante el uso de dichos procedimientos. Por otra parte, se ha 
comprobado que la biopsia de blastomera en el embrión en etapa de 8 células no afecta su viabilidad ni su capacidad de desarrollo(3-9).

El PGD se puede dividir en dos grandes modalidades según el método utilizado para el análisis del DNA, bien sea la Hibridización Fluorescente In Situ (FISH) o la Reacción en Cadena de Polimerasa (PCR). El FISH tiene como fin la identificación del sexo genético del embrión(10-11) y la presencia o no de anormalidades cromosómicas, principalmente aneuploidías (11-13). La PCR se puede utilizar también en la identificación del sexo genético (1), sin embargo, su principal uso tiene como objetivo la busqueda de genes únicos con capacidad de generar patologías conocidas como enfermedades monogénicas $(3,5,14)$.

El PGD puede ser una buena estrategia no sólo para evitar los embarazos con fetos genéticamente anormales, si no también para mejorar las tasas de implantación y/o embarazo principalmente en las mujeres de mayor edad. El estudio en embriones en el período preimplantación ha demostrado una alta incidencia de anomalías cromosómicas entre estos. Con el análisis de sólo 4 cromosomas $(\mathrm{X}, \mathrm{Y}, 18,16)$ se ha reportado un $33 \%$ de embriones anormales (15). En este mismo estudio se encontró que a pesar de que la incidencia de alteraciones cromosómicas era mayor $(42 \%)$ entre los embriones con morfología anormal (fragmentación $>15 \%$, blastómeras irregulares y multinucleadas, vacuolización marcada y granulación), esta seguía siendo importante entre aquellos con morfología normal (23\%). Además, se ha observado un aumento en la incidencia de alteraciones cromosómicas, principalmente aneuploidías, en relación con la edad de las pacientes sometidas a fertilización in vitro (FIV) (1519). En este grupo de mujeres las bajas tasas de implantación y embarazo parecen estar relacionadas con la calidad embrionaria, fenómeno demostrado por el aumento de estas tasas en los ciclos con donación de oocitos(20). El uso del PGD permitiría seleccionar los embriones sin alteraciones cromosómicas para ser transferidos, aumentando así las tasas de implantación y/o embarazo (12-13) y a la vez disminuyendo las tasas de aborto espontáneo en relación con el FIV.

A la luz de los conocimientos actuales aun quedan por resolver algunos interrogantes sobre la seguridad y la eficiencia del PGD para que este sea considerado como un procedimiento de rutina en la práctica de la reproducción asistida. Se han reportado aproximadamente 160 nacidos vivos luego de ser sometidos a un procedimiento de PGD (datos obtenidos del Segundo Simposio Internacional en Genética Preimplantación, Chicago, USA, Septiembre de 1997) y se ha observado que los embriones sometidos a el procedimiento de la biopsia de blastómera no ven afectado su desarrollo y viabilidad (3-9). Sin embargo la seguridad del procedimiento aun no está completamente comprobada pues es poca la información disponible en el momento sobre el seguimiento de los bebes nacidos luego de ser sometidos a un procedimiento de PGD en su etapa embrionaria temprana. Más allá de la seguridad del procedimiento, se requiere más claridad sobre su eficiencia. A pesar de que el riesgo de error parece ser relativamente bajo $(5-10 \%)$ hay reportes de fallas diagnósticas con el uso del $\operatorname{PGD}(3,9,21)$. Es por esto que la gran mayoría de los centros que lo ofrecen en el momento proponen la confirmación del diagnóstico genético con biopsia de vellosidades coriónicas o con amnioscentesis (22). Este aspecto se debe considerar al tratar con el paciente, haciendo énfasis en el carácter experimental aun del procedimiento.

No son de ignorar las implicaciones económicas del PGD. Este es un procedimiento complejo que requiere de un enfoque multidisciplinario y de tecnología de punta, aspectos que se traducen en un alto costo. A este costo elevado se suma el hecho de que tasas de embarazo entre 20-25\%, reportadas por la mayoría de los centros de FIV, parecen aun bajas para las parejas que requieren del PGD si se tiene en cuenta que la gran mayoría de ellas no tienen afectado su potencial fértil (23).

\section{Métodos para obtener el material genético}

Se han utilizado diversas maneras para obtener material genético que permita un análisis directo o indirecto del genoma embrionario preimplantación. Entre ellas se cuentan la biopsia de cuerpo polar, la biopsia de blastómera en el embrión en etapa de clivaje y la biopsia de trofoectodermo en el embrión en su etapa de blastocisto.

La biopsia de cuerpo polar se ha utilizado para descartar la existencia de alteraciones cromosómicas o de determinados genes mutantes en el oocito (24-26). De esta manera se logra cubrir una parte importante de las posibles alteraciones cromosómicas del embrión a formarse pues se considera que la mayor parte de ellas provienen de defectos a nivel del genoma del oocito (2729). Esta modalidad es claramente menos invasiva que la biopsia de blastómera, lo que parece traducirse en un aumento en las tasas de embarazo (30). Los oocitos sometidos a este procedimiento parecen mantener indemne su capacidad de dar origen a un embrión viable una vez fertilizados. Además, la biopsia de cuerpo polar permite un mayor lapso de tiempo para el análisis del material genético y la posterior toma de decisiones sobre la selección los embriones a transferir.

La biopsia de cuerpo polar tiene ciertas desventajas. En primer lugar no permite la detección de alteraciones cromosómicas de origen paterno y de alteraciones originadas en los procesos de fertilización (ej. polispermia) o embriogénesis. Obviamente, la determinación del sexo genético del embrión para la prevención de enfermedades ligadas al cromosoma X, está fuera de su alcance. Por último, esta técnica sólo permite un análisis indirecto del oocito y por ende del embrión resultante pues este se deduce del estudio del cuerpo polar que contiene un genotipo complementario al del oocito.

La biopsia de blastómera en el embrión en clivaje en su estado de 6 a 10 células (tercer día de cultivo) es la modalidad más frecuentemente utilizada para el PGD hoy en día. En esta etapa las blastómeras son aun células totipotenciales y la biopsia de 1 o 2 blastómeras no disminuye de manera drástica la masa celular del embrión (31-32). Por tal motivo el procedimiento no afecta la capacidad de desarrollo del mismo, lo que ha sido comprobado por varios autores $(3-9,33)$. Durante este 
tercer día de cultivo se realiza la biopsia de blastómera en la mañana, el análisis genético del material obtenido y la transferencia de los embriones seleccionados en la tarde. Algunos centros tienen como alternativa la transferencia de los embriones en la mañana siguiente (cuarto día de cultivo) (34) o la prolongación del cultivo hasta que el estos logren llegar a su estado de blastocisto.

La biopsia de blastómera presenta ciertas dificultades técnicas. Constituye un procedimiento con mayor grado de invasividad al embrión que la biopsia de cuerpo polar. Además, al igual que este último implica la manipulación de estructuras microscópicas que pueden perderse fácilmente en su procesamiento y que en ocasiones no aportan el material genético óptimo para el análisis $(15,35)$.

Otra desventaja de la biopsia de blastómera constituye la posibilidad de error generado por el análisis de una sola blastómera debido a la alta incidencia de mosaicismo (25$30 \%)$ en los embriones en clivaje (10, 12, 15, 36-39). Este margen de error puede ser disminuido más no abolido por la biopsia y análisis de 2 o más blastómeras. El significado del mosaicismo a nivel del embrión aun no está claramente determinado. Al parecer su importancia depende de la proporción de células cromosomicamente anormales en el embrión. Esto implica la posibilidad de desarrollo de fetos normales a partir de embriones con una pequeña cantidad de células anormales (15), debido a la menor capacidad proliferativa de las células anormales o a que un determinado porcentaje de células normales genera el desarrollo de un feto fenotípicamente normal. Otra posibilidad parece ser que el desarrollo normal o anormal depende del destino final de las células con alteraciones cromosómicas, la masa celular interna o el trofoectodermo(40).

Una tercera modalidad la constituye la biopsia de tejido extraembrionario realizada al blastocisto. Luego de 5 a 6 días de cultivo algunos el embriones logran llegar a esta etapa. Para este momento han surgido dos tipos celulares, la masa celular interna que dará origen al feto y la capa epitelial externa que dará origen al trofoectodermo. Es esta última estructura el lugar de donde se toma la biopsia, con el fin de evitar un procedimiento invasivo sobre las células encargadas de formar el feto (41-42). Por tal motivo la biopsia de trofoectodermo supone una menor agresión al verdadero tejido embrionario (la masa celular interna). Sin embargo, se debe tener en cuenta que con esta modalidad se tendrá un menor número de embriones disponibles para el diagnóstico pues se ha encontrado que sólo un 30-60\% de ellos logran llegar al estado de blastocisto en condiciones in vitro. Otro punto a tener en cuenta es el hecho de que el genoma de algunas células del trofoectodermo puede no ser representativo de las células en la masa celular interna. Esto se debe al fenómeno común de mosaicismo entre los embriones en etapa de clivaje $(15,40)$ y al destino final de aquellas células con anormalidades cromosómicas (masa celular interna vs. trofoectodermo).

\section{Técnicas de biopsia}

La técnica para biopsiar el embrión requiere de una cuidadosa micromanipulacion. Para la biopsia de blastómera se sostiene el embrión con una micropipeta de "hol- ding" con diámetro externo de 80 micras e interno de 20 micras. Luego se crea un orificio de aproximadamente 40 micras en la zona pelucida, mediante la instilación y posterior succión de Acido Tirodes (ph 2.4), utilizando una micropipeta de "drilling" con diámetro externo de 10 micras e interno de 8 micras. Finalmente se aspiran una o dos blastómeras con una micropipeta de biopsia con diámetro externo de 40 micras e interno de 34 micras (aproximadamente dos terceras partes del diámetro de la blastómera) (3, 43-44). Recientemente se ha utilizado tecnología con laser para realizar el orificio en la zona pelucida, sin embargo parece que su uso se relaciona con efectos adversos para el desarrollo embrionario y fetal (45).

La técnica de la biopsia de trofoectodermo se basa en la realización de una microincisión de la zona pelucida en el polo opuesto a la masa celular interna. A través de esta incisión ocurre una herniación del trofoectodermo que puede darse rápidamente o demorarse hasta 18-24 horas, momento en el cual se realiza la biopsia. Esta técnica permite obtener entre 10 y 30 células para el análisis genético (41).

\section{Hibridización Fluorescente In Situ (FISH)}

La Hibridización fluorescente in situ (FISH) en el PGD consiste en la identificación de cromosomas en el núcleo de una blastómera extendido y fijado en una laminilla de vidrio, mediante su hibridización con sondas de DNA marcadas fluorescentemente y específicas para ciertos cromosomas $(11,46)$. Cada sonda se adhiere al cromosoma al cual es afín y emite una señal fluorescente cuyo color difiere entre una y otra. De esta manera es posible determinar cuantos y cuales cromosomas contiene dicho núcleo. Hasta el momento se han producido sondas para los cromosomas $13,16,18,21,22$, X y Y, permitiendo así la detección de la aneuploidías más comunes $(3,11,15)$.

Actualmente el principal uso del FISH se da en la determinación del sexo genético del embrión para la prevención de enfermedades ligadas al cromosoma X (11, $23,47)$, tales como la distrofia muscular de Duchenne (48), la hemofilia A (49), el síndrome de cromosoma X frágil (50) y el síndrome de Lesch-Nyhan (51), entre otras. En esta situación específica, es un mejor método que la PCR ya que no sólo permite identificar la presencia del cromosoma X o Y, si no también las anormalidades en su número (10). Algunas técnicas complementarias se han utilizado para aumentar la proporción de embriones femeninos y por ende el número de embriones suceptibles a ser transferidos. Estas técnicas se basan en la manipulación de las proporciones de espermatozoides $\mathrm{X}$ y $\mathrm{Y}$ en la población total utilizada para la inseminación. Los mejores resultados se han logrado con el uso de la citometría de flujo (52), la cual permite la selección basándose en la diferencia del contenido de DNA de los espermatozoides X y Y $(2.8 \%)$. Poblaciones con una proporción de espermatozoides $\mathrm{X}$ del 80-90\% han sido reportadas.

Es posible que el uso sistemático del FISH para el análisis de los embriones obtenidos mediante FIV, pueda mejorar las tasas de implantación y embarazo en las mujeres mayores de 35 años $(10,12-13,15,53)$. Se ha 
reportado que la incidencia de aneuploidías en los embriones con morfología normal aumenta en proporción con la edad materna (15). Aunque aun no hay suficientes datos para comprobarlo, se presume que en las mujeres de mayor edad, la selección y transferencia de los embriones genéticamente normales permitiría mejorar las tasas de implantación y embarazo. Como se mencionó antes esta estrategia también podría disminuir las altas tasas de aborto espontáneo en este grupo de mujeres. Además de su uso para la determinación del sexo y detección de aneuploidías, es probable que el FISH permita el diagnóstico de translocaciones no balanceadas en embriones cuyos padres son portadores conocidos de una translocación balanceada (23).

El FISH es un procedimiento eficiente. Varios autores han reportado cifras de eficiencia que fluctuan alrededor del $90 \%(15,46-47,54-55)$. Sin embargo, aunque la contaminación no tiene mayor papel en los casos fallidos, hay otras cuasas de error que cabe mencionar. Las más importantes son la biopsia de blastómeras anucleadas, la presencia de micronúcleos, la pérdida de la blastómera durante el proceso de fijación, la ausencia de señal por falla en la hibridización y la sobreposición de la señal de los cromosomas.

\section{Reacción en Cadena de Plimerasa (PCR)}

La reacción en cadena de polimerasa (PCR) es al igual que el FISH, otra herramienta útil para el PGD. Con ella se logran amplificar secuencias conocidas de DNA que contienen mutaciones causantes de enfermedades hereditarias. De esta manera se obtienen millones de copias de esta sección específica del DNA a partir del material genético de una sola blastómera, permitiendo su análisis con electroforesis. A diferencia del FISH, el PCR permite el diagnóstico de patologías codificadas por genes únicos como la fibrosis quística $(4,6)$, la enfermedad de Tay-Sachs (5), la osteogénesis imperfecta (56) y el síndrome de Marfán (57) entre otras. Con su uso también es posible la determinación del sexo mediante la identificación de secuencias específicas de los cromosomas Y y X, aunque en este aspecto es inferior al FISH pues no permite el diagnóstico de alteraciones en el número de los mismos.

El PCR está sujeto a varias limitaciones en su aplicación para el PGD. La mayoría de ellas se relacionan con el uso de una sola célula como sustrato, lo que disminuye su eficiencia. La contaminación, el mal manejo de la muestra y el mosaicismo se cuentan entre las causas de falla en el diagnóstico $(3,9,21)$. Además, son de importancia la biopsia de blastómeras anucleares y la falla en la amplificación de las secuencias de DNA. La evaluación de defectos de un sólo gen con el uso una sola célula, implica que el material genético para el análisis se limite a lo amplificado a partir de 2 copias de la secuencia de DNA en estudio (2 alelos). En algunas ocasiones sólo una de estas 2 copias es amplificada, generando un mal diagnóstico por lo que se conoce como amplificación específica de alelo (58-59). Este fenómeno es particularmente importante cuando se trata de enfermedades autosómicas dominantes, ya que la falla de amplificación del alelo mutante hará parecer como no afectados a los embriones afectados.

Se han propuesto algunas modificaciones al PCR con miras aumentar su eficiencia y a disminuir su margen de error. La PCR fluorescente logra este cometido mediante la marcación con fluorescencia de las secuencias amplificadas y su identificación durante la electroforesis con la exitación mediante laser y no con el uso rutinario de bromuro de etidio (60). De esta manera se simplifica el proceso, se requieren menos ciclos de amplificación y se disminuyen los riesgos de contaminación. El principal inconveniente del PCR fluorescente es su alto costo.

El primer extension preamplification es otra opción desarrollada para mejorar la eficiencia del PCR en el PGD. Esta técnica permite amplificar la totalidad del contenido de DNA en la blastómera antes de amplificar secuencias específicas de DNA para el diagnóstico de defectos de genes únicos (61-63). De esta manera la cantidad de DNA disponible es mucho mayor y es posible el análisis simultáneo de varias patologías.

Por último, otro avance en el PGD conocido como el reciclaje celular permite el diagnóstico concomitante de aneuploidías, del sexo genético y de enfermedades monogénicas en el embrión a partir de una sola blastómera (64). Con esta técnica es posible el análisis de una célula fijada a una laminilla de vidrio, primero con PCR y luego con FISH. A pesar de que el PCR se realiza bajo condiciones subóptimas para permitir el posterior análisis con FISH, se ha reportado una eficiencia entre el 65$85 \%$ para esta modalidad diagnóstica (65-66).

\section{Conclusión}

El PGD es una ciencia en desarrollo que en sus primeras etapas ha demostrado ser viable y ha permitido beneficios claros a muchas parejas con riesgo de transmitir enfermedades genéticas a sus hijos. Un buen número de bebes sanos ha nacido como fruto de su aplicación. Sin embargo, no se puede desconocer la posibilidad del error diagnóstico asociado a esta técni$\mathrm{ca}$, con las consecuencias que este genera en un aspecto tan sensible.

El uso rutinario del PGD aun requiere de una mayor claridad en cuanto a su seguridad y eficacia. Un seguimiento a largo plazo de los pacientes permitirá definir sus posibles consecuencias sobre el desarrollo del individuo. Las parejas sometidas al PGD deben tener pleno conocimiento de que se trata aun de un procedimiento experimental y por ende se les debe recomendar la confirmación del diagnóstico con otros métodos como la biopsia de vellosidades coriónicas o la amnioscentesis.

Nuevos avances que permitan ampliar el espectro de análisis a más cromosomas y a un mayor número de enfermedades genéticas, es el curso a seguir. Además, una mejoría en las tasas de embarazo logradas con FIV contribuirá a la propagación del PGD.

Reconocimiento: Los autores agradecen la asesoría del Sr. Jose Roberto Alegretti, Jefe de la sección de embriología del Huntington Centro de Medicina Reproductiva (Sao Pablo, Brazil). 


\section{BIBLIOGRAFIA}

1. Handyside A, Kontogianni E, Hardy K, et al. Pregnancies from biopsied human preimplantation embryos sexed by Y-specific DNA amplification. Nature 1990; 344: 768-770.

2. Munné S, Cohen J. Preimplantation diagnosis in older patients-but of course. Human Reproduction 1997; 12: 413-414.

3. Grifo J, Tang Y, Munné S, Alikani M, Cohen J, Rosenwaks Z. Healthy deliveries from biopsied human embryos. Human Reproduction 1994; 9: 912-916.

4. Handyside A, Lesko J, Tarin J, Winston R, Hughes M. Birth of a normal girl after in vitro fertilization and preimplantation genetic testing. New England Journal of Medicine 1992;327: 905-909.

5. Gibbons W, Gitlin S, Lanzendorf S, Kaufmann R, Slotnick R, Hodgen G. Preimplantation genetic diagnosis for Tay Sachs disease: successful pregnancy after pre-embryo biopsy and gene amplification by polymerase chain reaction. Fertility and Sterility 1995; 63: 723-728.

6. Liu J, Lissens W, Silber S, Devroey P, Liebaers I, Van Steirteghem A. Birth after preimplantation diagnosis of the cystic fibrosis Delta F508 mutation by polymerase chain reaction in human embryos resulting from intracytoplasmic sperm injection with epididymal sperm. JAMA 1994; 272: 1858-1860.

7. Griffin D, Handyside A, Harper J, et al. Clinical experience with preimplantation diagnosis of sex by dual fluorescent in situ hybridization. Journal of Assisted Reproduction and Genetics 1994; 11: $132-143$

8. Veiga A, Santalo J, Vidal F, et al. Twin pregnancy after preimplantation diagnosis for sex selection - case report. Human Reproduction 1994; 1994:2156-2159.

9. Verlinsky Y, Handyside A, Grifo J, et al. Preimplantation diagnosis of genetic and chromosomal disorders. Journal of Assisted Reproduction and Genetics 1994; 11: 236-243.

10. Harper J, Coonen E, Handyside A, et al. Mosaicism of autosomes and sex chromosomes in morphologically normal, monospermic preimplantation human embryos. Prenat Diagn 1995; 15 41-49.

11. Munné S, Lee A, Rosenwaks Z, et al. Chromosome mosaicism in human embryos. Biol Reprod 1994; 51:373-379.

12. Munné S, Lee A, Rosenwaks Z, et al. Diagnosis of mayor chromosome aneuploidies in human preimplantation embryos. Human Reproduction 1993; 8: 2185-2191.

13. Verlinsky Y, Cieslak J, Freidine M, et al. Pregnancies following preconception diagnosis of common aneuploidies by fluorescent in-situ hybridization. Human Reproduction 1995; 10: 1923-1927.

14. Ray P, Winston R, Handyside A. Reduced allele dropout in single-cell analysis for preimplantation genetic diagnosis of cystic fibrosis. J Assist Reprod Genet 1996; 13: 104-106.

15. Munné S, Sultan K, Weier H, Grifo J, Cohen J, Rosenwaks Z. Assessment of numeric abnormalities of X, Y, 18 and 16 chromosomes in preimplantation human embryos before transfer. American Journal of Obstetrics and Gynecology 1995; 172: 1191-1201.

16. Munné S, Dailey T, Sultan K, Grifo J, Cohen J. The use of the first polar bodies for preimplantation diagnosis of aneuploidy. Human Reproduction 1995; 10: 1014-1020.

17. Munné S, Alikani M, Cohen J, Tompkins G, Grifo J. Implantation failure of morphologically normal human embryos is due largely to aneuploidy. Fertility and Sterility $1995 ; 64: 382-391$.

18. Munné S, Aliani M, Tomkin G, et al. Embryo morphology, developmental rates and maternal age are correlated with chromosome abnormalities. Fertility and Sterility 1995; 64: 382-391.

19. Dailey T, Dale B, Cohen J, Munné S. Association between nondisjunction and maternal age in meiosis-II human oocytes detected by FIS analysis. Am J Hum Genet 1996; 59: 2077-2078.

20. Sauer M, Paulson R, Lobo R. Reversing the natural decline in human fertility: an extended clinical trial of oocyte donation to women of advanced reproductive age. JAMA 1992; 268: 1275-1279.

21. Gibbons W, Gitlin S, Lanzendorf S. Strategies to respond to polymerase chain reaction deoxyribonucleic acid amplification failure in a preimplantation genetic diagnosis program. Am J Obstet Gynecol 1995; 172: 1088-1096.

22. Lissens W, Sermon K, Staessen C, et al. Review: Preimplantation diagnosis of inherited disease. J Inher Metab Dis 1996; 19: 709-723.
23. Lissens W, Sermon K. Preimplantation genetic diagnosis: current status and new developments. Human Reproduction 1997; 12: 1756 1761.

24. Strom C, Verlinsky Y, Milayeva S, et al. Preconception genetic diagnosis of cystic fibrosis. Lancet 1990; 336: 306-307.

25. Verlinsky Y, Kuliev A. Preimplantation diagnosis of common aneuploidies in infertile couples of advanced maternal age. Human Reproduction 1996; 11: 2076-2077.

26. Verlinsky Y, Ginsberg N, Lifchez A, et al. Analysis of the first polar body: preconception genetic diagnosis. Human Reproduction 1990; 5: $826-829$

27. Antonarakis S, Group DSC. Parental origin of the extra chromosome in trisomy 21 as indicated by analysis of DNA polymorphisms. New England Journal of Medicine 1991; 324: 872-876.

28. Fisher J, Harvey J, Lindenbaum R, Boyd P, Jacobs P. Molecular studies of trisomy 18. American Journal of Human Genetics 1993; 52 : 1139-1144.

29. Hassol T, Jacobs P, Leppert M, Sheldon M. Cytogenetic and molecular studies of trisomy 13. Journal of Medical Genetics 1987; 24: 725-732.

30. Verlinsky Y, Cieslak J, Ivakhnenko V, et al. Birth of healthy children after preimplantation diagnosis of common aneuploidies by polar body fluorescent in situ hybridization analysis. Fertility and Sterility 1996; 66: 126-129.

31. Tarin J, Handyside A. Embryo biopsy strategies for preimplantation diagnosis. Fertility and Sterility 1993; 59: 943-952.

32. Tarin J, Conaghan J, Winston R, et al. Human embryo biopsy on the 2nd day after insemination for preimplantation diagnosis: removal of a quarter of embryo retards cleavage. Fertility and Sterility 1992; 58 970-976.

33. Hardy $\mathrm{K}$, Martin $\mathrm{K}$, Leese $\mathrm{H}$, et al. Human preimplantation development in vitro is not adversely affected by biopsy at the 8-cell stage. Human Reproduction 1990; 5: 708-714.

34. Grifo J, Konstantinos G, Tang Y, C.Krey L. Successful outcome with day 4 embryo transfer after preimplantation diagnosis for genetically transmitted diseases. Human Reproduction 1998; 13: 1656-1659.

35. Grifo J, Tang Y, Munné S, Krey L. Update in preimplantation genetic diagnosis: successes, advances, and problems. Current Opinion in Obstetrics and Gynecology 1996; 8: 135-138.

36. Pellestor F, Dufour M, Arnal F, Humeau C. Direct assessment of the rate of chromosomal abnormalities in grade IV human embryos produced by in-vitro fertilization procedure. Human Reproduction 1994; 9: 293-302.

37. Plachot M, Junca A, Mandelbaum J, De Grouchi J, Salat-Baroux J, Cohen J. Chromosome investigations in early life: human preimplantation embryos. Human Reproduction 1987; 2: 29-35.

38. Bongso A, Fong C, Ng S, Ratman S, Lim J. Preimplantation genetics: chromosomes of fragmented human embryos. Fertility and Sterility 1991; 56: 66-70.

39. Delhanty J, Harper J, Ao A, et al. Multicolor FISH detects frequent chromosomal mosaicism and chaotic division in normal preimplantation embryos from fertile patients. Hum Genet 1997; 99: 755-760.

40. Edwards R, Beard H. Oocyte polarity and cell determination in early mammalian embryos. Mol Hum Reprod 1997; 3: 863-905.

41. Dokras A, Sargent I, Ross C, et al. Trophectoderm Biopsy in human blastocysts. Human Reproduction 1990; 5: 821-825.

42. Dokras A, Sargent I, Gardner R, et al. Human trophectoderm biopsy and secretion of chorionic gonadotropin. Human Reproduction 1991; 6: 1453-1459.

43. Chen S, Chao K, Wu M, Chen C, Ho H, Yang Y. The simplified twopipette technique is more efficient than the conventional three-pipette method for blastomere biopsy in human embryos. Fertility and Sterility 1998; 69: 569-575.

44. Grifo J, Tang Y, Cohen J, Gilbert F, Sanyal M, Rosenwaks Z. Ongoing pregnancy in a hemophilia carrier by embryo biopsy and simultaneous amplification of $\mathrm{X}$ and $\mathrm{Y}$ chromosome specific DNA from single blastomeres. JAMA 1992; 6: 727-729.

45. Licciardi F, Gonzalez A, Tang Y, Grifo J, Cohen J, Neev Y. Laser ablation of the mouse zona pellucida for blastomere biopsy. J Assist Reprod Genet 1995; 12: 462-466.

46. Harper J, Coonen E, Ramaekers F, et al. Identification of the sex of human preimplantation embryos in two hours using an 
improved spreading method and fluorescent in-situ hybridization (FISH) using directly labeled probes. Human Reproduction 1994; 9: $721-724$.

47. Staessen C, Van Assche E, Joris H, et al. Clinical experience of sex determination by fluorescent in-situ hybridization for preimplantation genetic diagnosis. Molecular Human Reproduction 1999; 5 : 382-389.

FÓRMULA:

PREMELLE GRAGEAS, PREMELLE PLUS, PREMELLE CÍCLICO incluye: estrógenos conjugados 0,625 mg y acetato de medroxiprogesterona (MPA), $2,5 \mathrm{mg} 65,0 \mathrm{mg}$, para administración oral. PREMELLE GRAGEAS PREMELLE PLUS Y PREMELLE CICLICO contiene una mezcla de estrógenos obtenidos exclusivamente de fuentes naturales, están compuestos por las sales sódicas de ésteres sulfato hidrosolubles de la estrona, la equilina y la 17 alfa-hidroxiequilina, además de cantidades más pequeñas de 17 alfa-estradiol, equilenina 17-alfa-dihidroequilenina, 17 beta-hidroequilina, 17 beta-dihidro- equilenina, 17 beta-estradiol y delta 8,9dehidroestrona.

\section{INDICACIOHES:}

1. Síntomas vasomotores moderados a severos asociados con una deficiencia de estrógenos.

2. Prevención y manejo de la Osteoporosis asociada con una deficiencia de estrógenos.

3. Vaginitis y Uretritis atrófica.

PREMELLE GRAGEAS, PREMELLE PLUS Y PREMELLE CÍCLICO se encuentra indicado en mujeres con útero intacto a fin de prevenir el riesgo de hiperplasia endometrial y cáncer endometrial asociado con la terapia de suplencia estrógenica. En el caso de pacientes histerectomizadas la sola terapia de Premarin es apropiada.

\section{CONTRAINDICACIONES:}

1. Cáncer conocido o sospechado de las mamas.

2. Neoplasia conocida o sospechada de estrógeno-dependiente

3. Embarazo conocido o sospechado

4. Hemorragia genital anormal no diagnosticada

5. Trastornos tromboflebíticos o tromboembólicos

6. Disfunción o enfermedad hepática

7. Hipersensibilidad a cualquiera de los componentes de PREMELLE GRAGEAS, PREMELLE PLUS Y PREMELLECÍCLICO

POSOLOGía Y adMINISTRación: La terapia continua con PREMELLE GRAGEAS, PREMELLE PLUS consiste en $0,625 \mathrm{mg}$ de estrógenos conjugados y $2,5 \mathrm{mg} 05 \mathrm{mg}$ de acetato de medroxiprogesterona para ser tomados simultáneamente una vez al día. La terapia continua con PREMELLE GRAGEAS, PREMELLE PLUS está indicada en mujeres con útero intacto para reducir el riesgo de hiperplasia endometrial y cáncer endometrial asociado con la terapia de suplencia estrogénica. La terapia sólo con Premarin solamente es apropiada para las pacientes histerectomizadas. La dosis inicial de 5,0 mg de acetato medroxiprogesterona es apropiada para aquellas pacientes en las cuales se deban lograr ciclos sin sangrado. Considérense la reducción de la dosis de $2.5 \mathrm{mg}$ después de alcanzar la amenorrea o dentro de los 12 meses de iniciada la terapia. Si un sangrado ocurre después de la reducción del acetato de medroxiprogesterona a $2.5 \mathrm{mg}$ considere reiniciar el régimen de $5,0 \mathrm{mg}$. La dosis inicial habitual con PREMELLE CONTINUO, Y PREMELLE CíCLICO es de $5,0 \mathrm{mg}$.

Adicionalmente se deben tener en cuenta los siguientes factores cuando se ajuste la dosis del acetato de medroxiorogesterona: historia médica de la paciente, presencia de eventos adversos, resultado de laboratorio y exámenes físico y ginecológico. Las pacientes deben ser evaluadas nuevamente en intervalos regulares.

\section{ADVERTENCIAS:}

Algunos estudios sugirieron un posible aumento de la incidencia de cáncer de mama en aquellas mujeres que reciben dosis elevadas de estrógenos durante un periodo prolongado. Sin embargo, la mayoria de los estudios no corroboraron esta asociación con las dosis habitualmente utilizadas para la terapia de suplencia estrogénica. Las mujeres que reciben este tratamiento deben ser evaluadas periódicamente con exámenes mamarios y recibir instrucciones para que puedan llevar a cabo el autoexamen mamario. Se reportó un riesgo aumentado de enfermedad de la vesícula biliar en las mujeres posmenopáusicas tratadas con estrógenos. Las dosis de PREMELLE GRAGEAS, PREMELLEPLUS Y PREMELLE CÍCLICO usadas en la terapia de suplencia hormonal no deben ser mayores que las dosis recomendadas. El uso de PREMELLE GRAGEAS, PREMELLE PLUS Y PREMELLE CÍCLICO durante la menopausia se asoció con aumentos de la presión sanguínea. Se recomienda el control periódico de la presión arterial.

No se documentó un riesgo aumentado de tromboflebitis o de accidentes tromboembólicos en las mujeres que reciben una terapia de suplencia estrogénica. Sin embargo, no se cuenta con una cantidad suficiente de información para establecer con certeza dicho riesgo en las mujeres con antecedentes de enfermedad tromboembólica. El médico debe mantenerse alerta a fin de detectar las manifestaciones más tempranas de Ios trastornos trombóticos (tromboflebitis, accidentes cerebrovasculares, embolia pulmonar y trombosis retiniana). Ante la aparición 0 la sospecha de cualquiera de estos problemas se encuentra indicada la 'interrupción inmediata de PREMELLE GRAGEAS, PREMELLE PLUS Y PREMELLE CíCLICO y suministrar tratamiento apropiado a la paciente.

Se recomienda interurmpir el tratamiento con PREMELLE GRAGEAS, PREMELLEPLUS Y PREMELLE CÍCLICO hasta esperar los resultados del examen médico ante la aparición de una pérdida parcial o completa de la visión o ante la instalación brusca de proptosis, diplopia o migraña. Si el examen oftalmológico revela edema de papila o lesiones vasculares retinianas, el tratamiento debe ser definitivamente interrumpido.

USO DURANTE EL EMBARAZO: PREMELLE GRAGEAS, PREMELLE PLUS Y PREMELLE CÍCLICO no deben ser utilizados durante el embarazo.

\section{PRECAUCIONES:}

Es importante obtener una ínformación médica y familiar completa antes de iniciar cualquier tratamiento con estrógenos. Loe exámenes físicos pretratamiento y periódicos durante el tratamiento deben consignar cualquier dato anormal relacionado con la presión sanguínea, las mamas, el abdomeny los pelvianos e incluir un extendido con la técnica de Papanicolaou.

\section{PRESENTACIONES:}

PREMELLE GRAGEAS: Caja x 28 grageas de Estrógeno Naturales Conjugados $0,625 \mathrm{mg}$ y Acetato de Medroxiprogesterona 2,5 mgReg. INVIMA M-010941.

PREMELLE PLUS Grageas: Caja de 28 grageas de Estrógenos Naturales Conjugados $0,625 \mathrm{mg}$ y Acetato de Medroxiprogesterona 5,0 mg Reg. INVIMAM- 010940.

PREMELLE CÍCLICO: Caja x 28 grageas: 14 grageas de Estrógenos Naturales Conjugados 0,625 mg y 14 grageas de Estrógenos Naturales Conjugados $0,625 \mathrm{mg}+$ Acetato de Medroxiprogesterona $5 \mathrm{mg}$. Reg. INVIMA M-011988

REFERENCIAS:

1. Archer DF, Pickar JH, Bottiglioni F for The Menopause Study Group. Obstetric Gynecol 83:686-692

2. Woodruff JD, Pickar JH,for The Menopause Study Group, Am J Obstetric Gynecol 70:1213-1223, 1994

48. Liu J, Lissens W, Van Broeckhoven C, et al. Normal pregnancy after preimplantation diagnosis of a dystrophic gene deletion. Prenat Diagn 1995; 15: 351-358.

49. Boada M, Carrera M, De La Iglesia C, Sandalinas M, Barri P, Veiga A. Successful use of a laser for human embryo biopsy in preimplantation genetic diagnosis: report of two cases. Journal of Assisted Reproduction and Genetics 1998; 15: 302-307.

50. Dreesen J, Geraedts J, Dumoulin J, et al. RS46 (DXS548) genotyping of reproductive cells: approaching preimplantation testing of the fragile-X syndrome. Hum Genet 1995; 96: 323-329.

51. Ray P, Winston R, Handyside A. Single cell analysis for diagnosis of cystic fibrosis and Lesch-Nyhan syndrome in human embryos before implantation. In: Miami Bio/Technology Short Reports: Proceedings of the $1994 \mathrm{Miami} / \mathrm{Technology}$ European Symposium, Advances in Gene Technology: Molecular biology and Human Genetic Disease, Miami Bio/Technology European Symposium, Miami, 1994. Vol. 5.

52. Levinson $\mathrm{G}$, Keyvanfar K, Wu J, et al. DNA-based X-enriched sperm separation as an adjunct to preimplantation genetic testing for the prevention of X-linked disease. Human Reproduction 1995; 10: 979-982.

53. Munné S, Benadiva C, Cohen J, Grifo J. Preimplantation diagnosis of aneuploidy in women of 40 years or older (Abstract). Fertility and Sterility 1995; 64:O-076.

54. Munné S, Grifo J, Cohen J, et al. Chromosome abnormalities in human arrested preimplantation embryos: a multiple-probe FISH study. Am J Hum Genet 1994; 55: 150-159.

55. Coonen E, Dumoulin J, Dreesen C, et al. Clinical application of FISH for sex determination of embryos in preimplantation diagnosis of $\mathrm{X}$ linked diseases. J Assist Reprod Genet 1996; 10: 82-90.

56. Lida T, Suzumori K, Ikuta K, et al. Identification of a Gly 862 to Ser substitution in the type I collagen gene from a single spermatozoon. Mol Hum Reprod 1996; 2:131-134.

57. Harton G, Tsipouras P, Sisson M, et al. Preimplantation genetic testing for Marfan syndrome. Mol Hum Reprod 1996; 2: 713-715.

58. Pickering S, Muggleton-Harris A. Reliability and accuracy of polymerase chain reaction amplification of two unique target sequences frombiopsies of cleavage-stage and blastocyst-stage human embryos. Human Reproduction 1995; 10: 1021-1029.

59. Eldadah D, Grifo J, Dietz H. Marfan syndrome as a paradigm for transcript-targeted preimplantation diagnosis of heterozygous mutations. Nature Medicine 1995; 8: 798-803.

60. Hattori M, Yoshioka K, Sakaki Y. Highly-sensitive fluorescent DNA sequencing and its application for detection and mass-screening of point mutations. Electrophoresis 1992; 13: 560-565.

61. Kristjansson K, Chong S, Van den Veyver I, Subramanian S, Snabes M, Hughes M. Preimplantation single cell analysis of dystrophin gene deletions using whole genome amplification. Nature Genetics 1994; 6: 19-23.

62. Xu K, Tang Y, Grifo J, Rosenwaks Z, Cohen J. Primer extension preamplification for detection of multiple genetic loci from single human blastomeres. Human Reproduction 1993; 8: 2206-2210.

63. Zhang L, Cui X, Schmitt K, Hubert R, Navidi W, Arnheim N. Whole genome amplification from a single cell: implications for genetic analysis. Proceedings of the National Academy of Sciences of the USA 1992; 89: 5847-5851.

64. Thornhill A, Holding C, Monk M. Recycling the single cell to detect specific chromosomes and to investigate specific gene sequences. Human Reproduction 1994; 9: 2150-2155.

65. Thornhill A, Monk M. Cell recycling of a single human cell for preimplantation diagnosis of X-linked disease and dual sex determination. Mol Hum Reprod 1996; 2: 285-289.

66. Rechitsky S, Freidine M, Verlinsky Y, et al. Allele dropout in sequential PCR and FISH analysis of single cells (cell recycling). J Assist Reprod Genet 1996; 13: 115-124. 